\title{
Time delay in the Einstein-Straus solution
}

\author{
Kheir-Eddine Boudjemaa1, Mourad Guenouche and Sami R. Zouzou
}

Laboratoire de Physique Théorique, Département de Physique, Faculté des Sciences, Université Mentouri-Constantine, Algeria

boudjemaa_kh2006@yahoo.fr

guenouchemourad@live.com

szouzou2000@yahoo.fr

\begin{abstract}
We compute the time delay of strong lensing in the framework of the Einstein-Straus solution. We compare the theory to the observational bound on the time delay of the lens SDSS $\mathrm{J} 1004+4112$.
\end{abstract}

Keywords: Cosmological parameters. Lensing. Time delay. Einstein-Straus solution PACS: 98.80.Es, 98.80.Jk

\section{Introduction}

Before the work of Rindler and Ishak [1], the general believe was that the deflexion angle of light passing near an isolated static and spherically symmetric mass is independent of the cosmological constant. This believe was based on the argument that the cosmological constant disappears from the geodesic equation for massless particles. In september 2007, Rindler and Ishak [1] corrected this believe. They pointed out that it is not sufficient to consider the geodesic equation but also the metric itself must be considered. In the work of Rindler and Ishak the source emitting the light and the Earth were supposed to be at rest with respect to the lens.

\footnotetext{
${ }^{1}$ also at Centre Universitaire de Khenchela, Algeria.
} 
Also all the masses, including those of the Earth and of the source, were neglected, except the mass of the lens. Since then, there is a rich controversy about whether or not a cosmological constant modifies the bending of light near an isolated spherical mass. Sereno [2, 3], Schücker [4, 5], Miraghaei and Nouri-Zonoz [6], Kantowski, Chen and Dai [7, confirm Rindler and Ishak's result, while Khriplovich and Pomeransky [8], Park [9], Gibbons, Warnick and Werner [10], Simpson, Peacock and Heavens [11] contradict Rindler and Ishak's findings.

Recently, Schücker [12] redid the calculations of the bending of light by a spherically symmetric mass distribution, which is taken to be a cluster of galaxies by relaxing all the previous hypotheses except that of sphericity: the observer and the source are allowed to move with respect to the cluster, the masses of the other clusters are included in the form of a homogeneous isotropic dust and the observer as well as the source are taken comoving with respect to the dust. The appropriate framework of this computation is the Einstein-Straus solution [13, 14] that matches the Kottler solution at the inside of the Schücking radius with the Friedmann solution at the outside. Einstein and Straus' first motivation was to explain why the cosmic expansion does not affect smaller length scales such as planetary and atomic systems. Schücker's calculations confirm previous calculations by Ishak, Rindler, Dossett, Moldenhauer and Allison [15]: Taking into account realistic cosmic velocities attenuates the effect of the cosmological constant on the bending of light without however cancelling it. Although there has never been a claim that the time delay was independent of a cosmological constant, it is also interesting to do the computation of the time delay in the framework of the Einstein-Straus solution.

We will use the same units as in reference [12]: astrometers (am), astroseconds (as), astrograms (ag)

$$
\begin{aligned}
\mathrm{am} & =1.30 \cdot 10^{26} \mathrm{~m}=4221 \mathrm{Mpc} \\
\mathrm{as} & =4.34 \cdot 10^{17} \mathrm{~s}=13.8 \mathrm{Gyr} \\
\mathrm{ag} & =6.99 \cdot 10^{51} \mathrm{~kg}=3.52 \cdot 10^{21} \mathrm{M}_{\odot},
\end{aligned}
$$

where $\mathrm{M}_{\odot}$ denotes one solar mass. In these units

$$
\mathrm{c}=1 \mathrm{am} \mathrm{as}^{-1}, 8 \pi G=1 \mathrm{am}^{3} \mathrm{as}^{-2} \mathrm{as}^{-1}, H_{0}=1 \mathrm{as}^{-1}, \hbar=3.86 \cdot 10^{-121} \mathrm{am}^{2} \mathrm{as}^{-1} \mathrm{ag} .
$$

For spatially flat universes, to which we will restrict ourselves in the following, we may set the scale factor today $a_{0}=1$.

\section{The Einstein-Straus solution with a cosmological constant}

We will consider hereafter the Einstein-Straus solution [13, 14] generalized to include the cosmological constant [16] but restrict ourselves to spatially flat universes. We will need the Jacobian of the transformation passing between the Friedmann and Schwarzschild coordinates to calculate the geodesics of photons. Let us quote the results obtained by Schücker [12]. Let $(T, r, \theta, \varphi)$ and $(t, \chi, \theta, \varphi)$ stand respectively for Kottler and Friedmann coordinates. The Kottler metric

$$
d s^{2}=B(r) d T^{2}-B(r)^{-1} d r^{2}-r^{2} d \Omega^{2},
$$

with

$$
B(r)=1-\frac{2 G M}{r}-\frac{\Lambda}{3} r^{2},
$$

prevails inside a vacuole of radius $r_{S c h u ̈}(T), r<r_{S c h \ddot{u}}$. The Friedmann spatially flat metric is given by:

$$
d s^{2}=d t^{2}-a(t)^{2}\left(d \chi^{2}+\chi^{2} d \Omega^{2}\right)
$$


with the scale factor $a(t)$ determined by the first order Friedmann equation

$$
\frac{d a}{d t}=\sqrt{A / a+\Lambda a^{2} / 3},
$$

where

$$
A=a_{0}^{3} \rho_{\text {dust } 0} / 3=1-\frac{\Lambda}{3},
$$

prevails outside the vacuole $\chi \geq \chi_{S c h \ddot{u}}$. It is worthwhile to notice that due to (6), the scale factor is strictly monotonic. The two solutions are glued together at the constant Schücking radius $\chi_{S c h u ̈}$

$$
r_{S c h \ddot{u}}(T):=a(t) \chi_{S c h \ddot{u}} .
$$

By taking into account the fact that the central mass must be equal to the dust density times the volume of the ball with Schücking radius $r_{S c h \ddot{u}}$

$$
\begin{aligned}
A & =\frac{2 M}{8 \pi \chi_{S c h u ̈}^{3}}=\frac{2 G M}{\chi_{S c h \ddot{u}}^{3}} \\
B\left(r_{S c h \ddot{u}}\right) & =: \quad B_{S c h \ddot{u}}=1-\frac{A}{a} \chi_{S c h \ddot{u}}^{2}-\frac{\Lambda}{3} a^{2} \chi_{S c h \ddot{u}}^{2} .
\end{aligned}
$$

It is also useful to introduce $C_{S c h u ̈}$

$$
C_{S c h \ddot{u}}:=\sqrt{1-B_{S c h \ddot{u}}} .
$$

Schücker [12] computed the Jacobian of the coordinate transformation at the Schücking radius and also the inverse of the Jacobian (corresponding to the Jacobian of the inverse coordinate transformation $(t, \chi) \rightarrow(T, r))$ with the results

$$
\begin{array}{rlrl}
\left.\frac{\partial t}{\partial T}\right|_{S c h \ddot{u}} & =1, & \left.\frac{\partial t}{\partial r}\right|_{S c h \ddot{u}} & =-\frac{C_{\text {Schü }}}{B_{\text {Schü }}} \\
\left.\frac{\partial \chi}{\partial T}\right|_{S c h \ddot{u}}=-\frac{C_{S c h \ddot{u}}}{a}, & \left.\frac{\partial \chi}{\partial r}\right|_{S c h \ddot{u}} & =\frac{1}{a B_{S c h \ddot{u}}},
\end{array}
$$

and

$$
\begin{aligned}
\left.\frac{\partial T}{\partial t}\right|_{S c h \ddot{u}} & =\frac{1}{B_{S c h \ddot{u}}}, & \left.\frac{\partial T}{\partial \chi}\right|_{S c h \ddot{u}} & =a \frac{C_{S c h \ddot{u}}}{B_{S c h \ddot{u}}} \\
\left.\frac{\partial r}{\partial t}\right|_{S c h \ddot{u}} & =C_{S c h \ddot{u}}, & \left.\frac{\partial r}{\partial \chi}\right|_{S c h \ddot{u}} & =a .
\end{aligned}
$$

In the following we also need to pass between Kottler time $T$ and Friedmann time $t$ at the Schücking radius. To this end we will use the result also obtained by Schücker [12]

$$
\left.\frac{d t}{d T}\right|_{S c h \ddot{u}}=B_{S c h \ddot{u}} .
$$

\section{$3 \quad$ Integrating the geodesics of light}

We have the following situation: a first photon is emitted by the source, a quasar, at a time $t_{S}^{\prime}$ and follows an upper straight line trajectory until its arrival at a time $t_{S c h u ̈ S}^{\prime}$ on the Schücking sphere in the half space containing the source. It is then bent inside the Schücking radius, until 
it emerges from the Schücking sphere in the half space containing the Earth at time $t_{S c h u ̈ E}^{\prime}$, then follows a straight line until its arrival on Earth at time $t_{0}^{\prime}=0$. A second photon is emitted by the quasar at a time $t_{S}$ follows a lower straight line trajectory, arrives on the Schücking sphere in the half space containing the source at time $t_{S c h \ddot{u} S}$, it is then bent inside the Schücking sphere and emerges from the Schücking sphere in the half space containing the Earth at time $t_{S c h u ̈ E}$, follows again a straight line until its receipt on Earth at the same time as the first photon $t_{0}=t_{0}^{\prime}=0$. We will here be interested in the computation of the time delay $t_{S}-t_{S}^{\prime}$.

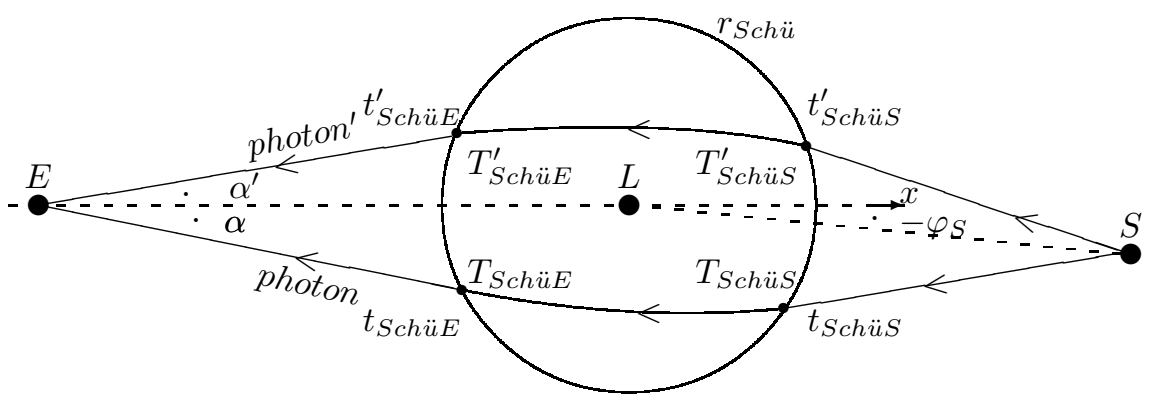

Figure 1: Two light rays emitted by a source $S$, bent inside the Schücking sphere and finally received at Earth $E$. The travel times of the two photons differ, giving rise to a time delay.

Let us first integrate the first order Friedmann solution (6) for the scale factor $a(t)$ in the spatially flat case with cosmological constant $\Lambda$ and dust density $\rho_{\text {dust } 0}=(3-\Lambda)$ (we will take the experimentally favored value $\left.\Lambda=0.77 \cdot 3 \mathrm{am}^{-2} \pm 20 \%\right)$ with final condition $a(0)=1$. One may show that (6) is equivalent to the Friedmann second order equation for $a(t)$

$$
\frac{2}{a} \frac{d^{2} a}{d t^{2}}+\frac{1}{a^{2}}\left(\frac{d a}{d t}\right)^{2}=\Lambda
$$

with final conditions

$$
\left.\frac{d a}{d t}\right|_{t=0}=1, \quad a(0)=1 .
$$

The integration of (6) or (15) may be done analytically with the result

$$
a(t)=\left(\frac{1-\Lambda / 3}{\Lambda / 3}\right)^{1 / 3}\left[\sinh ^{2}\left(\frac{3}{2} \sqrt{\frac{\Lambda}{3}} t+\operatorname{arcsinh}\left(\frac{\Lambda / 3}{1-\Lambda / 3}\right)\right)\right]^{1 / 3} .
$$

We will also need to solve the equation

$$
\frac{d \chi}{d t}=-\frac{1}{a}
$$

for various final conditions, with $\chi$ having the meaning of a geodesic distance, not to be confused with a luminosity distance. Since these are the final conditions, at the arrival on Earth, which are known, we will proceed backwards in time in three steps: we will determinate $t_{S c h u ̈ E}^{\prime}$ and

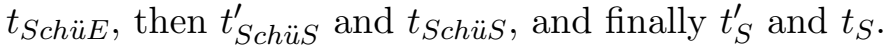


step 1: Determination of $t_{S c h u ̈ E}^{\prime}$ and $t_{S c h u ̈ E}$.

Here we will be interested in the photon trajectory between the Earth and the Schücking sphere with the Friedmann metric. The non vanishing Christoffel symbols of the Friedmann metric in the plane $\theta=\pi / 2$ are given by

$$
\begin{array}{ll}
\Gamma_{\chi \chi}^{t}=a a_{t}, & \Gamma_{\varphi \varphi}^{t}=a a_{t} \chi^{2} \\
\Gamma_{\varphi \varphi}^{\chi}=-\chi, & \Gamma_{t \chi}^{\chi}=a_{t} / a, \\
\Gamma_{t \varphi}^{\varphi}=a_{t} / a, & \Gamma_{\chi \varphi}^{\varphi}=1 / \chi,
\end{array}
$$

and the geodesic equation reads

$$
\begin{aligned}
\ddot{t}+a a_{t} \dot{\chi}^{2}+a a_{t} \chi^{2} \dot{\varphi}^{2} & =0, \\
\ddot{\chi}+2 \frac{a_{t}}{a} \dot{t} \dot{\chi}-\chi \dot{\varphi}^{2} & =0, \\
\ddot{\varphi}+2 \frac{a_{t}}{a} \dot{t} \dot{\varphi}+\frac{2}{\chi} \dot{\chi} \dot{\varphi} & =0,
\end{aligned}
$$

with final conditions at $p=0$

$$
\begin{array}{lll}
t=0, & \chi=\chi_{E}, & \varphi=\pi \\
\dot{t}=1, & \dot{\chi}=\cos \alpha^{\prime}, & \dot{\varphi}=\frac{\sin \alpha^{\prime}}{\chi_{E}},
\end{array}
$$

for the upper trajectory photon, where we use the fact that the physical angle $\alpha^{\prime}$ coincides with the coordinate angle $\arctan (|\chi \dot{\varphi} / \dot{\chi}|)$.

For the lower trajectory photon, the final conditions differ

$$
\begin{array}{lll}
t=0, & \chi=\chi_{E}, & \varphi=-\pi \\
\dot{t}=1, & \dot{\chi}=\cos \alpha, & \dot{\varphi}=-\frac{\sin \alpha}{\chi_{E}} .
\end{array}
$$

The solution of the geodesic equation is

$$
\dot{t}=\frac{1}{a}, \quad \frac{\chi_{p}^{\prime}}{\chi}=\sin \left(\varphi-\alpha^{\prime}\right), \quad \dot{\varphi}=\frac{\chi_{p}^{\prime}}{a^{2} \chi^{2}},
$$

where $\chi_{p}^{\prime}$, the would be peri-lens, is given by

$$
\chi_{p}^{\prime}=\chi_{E} \sin \alpha^{\prime}
$$

for the upper trajectory photon and

$$
\dot{t}=\frac{1}{a}, \quad \frac{\chi_{p}}{\chi}=-\sin (\varphi+\alpha), \quad \dot{\varphi}=-\frac{\chi_{p}}{a^{2} \chi^{2}}
$$


where $\chi_{p}$, the would be peri-lens, is given by

$$
\chi_{p}=\chi_{E} \sin \alpha,
$$

for the lower trajectory photon.

The polar angles $\varphi_{S c h \ddot{u} E}^{\prime}$ and $\varphi_{S c h \ddot{u} E}$ at which the lower and the upper trajectory photons emerge from the Schücking sphere are given respectively by

$$
\begin{gathered}
\varphi_{\text {SchüE }}^{\prime}=\pi-\arcsin \left(\frac{\chi_{p}^{\prime}}{\chi_{S c h \ddot{u}}}\right)+\alpha^{\prime}, \\
\varphi_{S c h \ddot{u} E}=-\pi+\arcsin \left(\frac{\chi_{p}}{\chi_{S c h \ddot{u}}}\right)-\alpha,
\end{gathered}
$$

where $\chi_{S c h \ddot{u}}$, the Schücking radius, is given by

$$
\chi_{S c h \ddot{u}}=\left(\frac{M}{4 \pi(1-\Lambda / 3)}\right)^{1 / 3}
$$

where we make use of (7) together with the fact that we work in system of units such that $A=1-\Lambda / 3$. It is easy to see using elementary Euclidean geometry that the geodesic distance $\chi_{S c h u ̈ E, E}^{\prime}$ between the upper trajectory photon when it emerges from the Schücking sphere and the Earth is given by

$$
\chi_{S c h \ddot{u} E, E}^{\prime}=\sqrt{\chi_{E}^{2}+\chi_{S c h u ̈}^{2}+2 \chi_{E} \chi_{S c h \ddot{u}} \cos \varphi_{S c h \ddot{U} E}^{\prime}},
$$

where $\chi_{E}$ is the Earth-lens geodesic distance.

In the same way the geodesic distance $\chi_{S c h u ̈ E, E}$, between the lower trajectory photon when it emerges from the Schücking sphere and the Earth is given by an analogous expression

$$
\chi_{S c h \ddot{u} E, E}=\sqrt{\chi_{E}^{2}+\chi_{S c h u ̈}^{2}+2 \chi_{E} \chi_{S c h \ddot{u}} \cos \varphi_{S c h \ddot{u} E}},
$$

The Earth-Lens geodesic distance $\chi_{E}$ may be deduced from the knowledge of the redshift $z_{E}$ according to the scheme

$$
z_{E} \rightarrow a_{E}=\frac{1}{1+z_{E}} \rightarrow \widetilde{t}_{E}:=\widetilde{t}\left(a_{E}\right) \rightarrow \chi\left(\widetilde{t}_{E}\right)=: \chi_{E}
$$

where $\widetilde{t}(a)$ denotes the inverse of the scale factor $a(t)$, where $\chi$ is the solution of the first order differential equation (18), subject to the initial condition $\chi(0)=0$, meaning that the photon reaches the Earth today at $t_{0}=0$. Moreover, since the scale factor $a$ is strictly positif, $\chi$ is a strictly decreasing function and thus injective. Therefore, $\chi(t)$ can be inverted to give $t$ in term of $\chi$.

The knowledge of $z_{E}$ allows to determine $a_{E}$, then one deduces the corresponding time $\widetilde{t}_{E}$. Injecting in $\chi(t)$, one finally deduces $\chi_{E}$. This is the same procedure that will be used to deduce $\chi_{S}$

$$
z_{S} \rightarrow a_{S}=\frac{1}{1+z_{S}} \rightarrow \widetilde{t}_{S}:=\widetilde{t}\left(a_{S}\right) \rightarrow \chi\left(\widetilde{t}_{S}\right)=: \chi_{S}
$$

On the other hand, the geodesic distance between the upper trajectory photon and the Earth in the time interval from the crossing of the Schücking sphere in the half space 
containing the Earth $t_{S c h u ̈ E}^{\prime}$ until its arrival at Earth $t_{0}^{\prime}=0$ is governed by equation (18) with the final condition

$$
\chi(0)=0
$$

meaning that at $t_{0}^{\prime}=0$ the photon reaches the Earth. From (18) and (34), one deduces that

$$
\int_{0}^{\chi_{S c h u ̈ E, E}^{\prime}} d \chi=-\int_{0}^{t_{S c h u ̈ E}^{\prime}} \frac{1}{a(t)} d t
$$

i.e.,

$$
\chi_{S c h \ddot{u} E, E}^{\prime}=-\int_{0}^{t_{S c h u ̈ E}^{\prime}} \frac{1}{a(t)} d t=\int_{t_{S c h u ̈ E}^{\prime}}^{0} \frac{1}{a(t)} d t .
$$

Then, comparing with equation (30), one gets

$$
\sqrt{\chi_{E}^{2}+\chi_{S c h \ddot{u}}^{2}+2 \chi_{E} \chi_{S c h \ddot{u}} \cos \varphi_{S c h \ddot{u} E}^{\prime}}=\int_{t_{S c h \ddot{u} E}^{\prime}}^{0} \frac{1}{a(t)} d t .
$$

Equation (37) may then be used to deduce $t_{S c h u ̈ E}^{\prime}$.

The geodesic distance between the lower trajectory photon and the Earth in the time interval from the crossing of the Schücking sphere in the half space containing Earth, $t_{S c h u ̈ E}$, until its arrival at Earth $\left(t_{0}=t_{0}^{\prime}=0\right)$, is also governed by (18) with the final condition (34). In an analogous way, one gets

$$
\chi_{S c h u ̈ E, E}=\int_{t_{S c h u ̈ E}}^{0} \frac{1}{a(t)} d t=\int_{t_{S c h u ̈ E}}^{0} \frac{1}{a(t)} d t .
$$

Then, comparison with equation (31) gives

$$
\sqrt{\chi_{E}^{2}+\chi_{S c h \ddot{u}}^{2}+2 \chi_{E} \chi_{S c h \ddot{u}} \cos \varphi_{S c h u ̈ E}}=\int_{t_{S c h u ̈ E}}^{0} \frac{1}{a(t)} d t,
$$

which may serve to deduce $t_{S c h u ̈ E}$.

However, we find it more reliable to proceed in a different way: we compute $t_{S c h u ̈ E}$ by difference with $t_{S c h u ̈ E}^{\prime}$. Combining (37) and (39), one gets

$$
\chi_{S c h \ddot{u} E, E}-\chi_{S c h \ddot{u} E, E}^{\prime}=\int_{t_{S c h u ̈ E}}^{0} \frac{1}{a(t)} d t-\int_{t_{S c h u ̈ E}^{\prime}}^{0} \frac{1}{a(t)} d t=\int_{t_{S c h u ̈ E}}^{t_{S c h u ̈ E}^{\prime}} \frac{1}{a(t)} d t .
$$

Since $a(t)$ does vary significantly only on cosmological time scales and since $\left|t_{\text {SchüE }}^{\prime}-t_{S c h \ddot{u} E}\right|$ is very much smaller than cosmological time scales then

$$
\int_{t_{S c h u ̈ E}}^{t_{S c h \ddot{u} E}^{\prime}} \frac{1}{a(t)} d t \simeq \frac{t_{S c h \ddot{u} E}^{\prime}-t_{S c h \ddot{u} E}}{a\left(t_{S c h u ̈ E}^{\prime}\right)} .
$$

On the other hand, combining (30) and (31)

$$
\begin{aligned}
\chi_{S c h \ddot{u} E, E}-\chi_{S c h \ddot{u} E, E}^{\prime}= & \sqrt{\chi_{E}^{2}+\chi_{S c h \ddot{u}}^{2}+2 \chi_{E} \chi_{S c h \ddot{u}} \cos \varphi_{S c h u ̈ E}} \\
- & \sqrt{\chi_{E}^{2}+\chi_{S c h u ̈}^{2}+2 \chi_{E} \chi_{S c h \ddot{u}} \cos \varphi_{S c h u ̈ E}^{\prime}} .
\end{aligned}
$$


But

$$
\begin{aligned}
\cos \varphi_{S c h \ddot{u} E}^{\prime} & =-\cos \left(-\arcsin \left(\frac{\chi_{E} \sin \alpha^{\prime}}{\chi_{S \operatorname{sh} \ddot{u}}}\right)+\alpha^{\prime}\right) \\
& \simeq-\cos \left(-\frac{\chi_{E} \sin \alpha^{\prime}}{\chi_{S c h \ddot{u}}}+\alpha^{\prime}\right) \\
& \simeq-1+\frac{1}{2}\left(\frac{\chi_{E}-\chi_{S c h \ddot{u}}}{\chi_{S c h \ddot{u}}}\right)^{2} \alpha^{\prime 2},
\end{aligned}
$$

and similarly

$$
\cos \varphi_{S c h \ddot{u} E} \simeq-1+\frac{1}{2}\left(\frac{\chi_{E}-\chi_{S c h \ddot{u}}}{\chi_{S c h \ddot{u}}}\right)^{2} \alpha^{2},
$$

and then one gets up to second order in the physical angles $\alpha$ and $\alpha^{\prime}$

$$
\chi_{S c h \ddot{u} E, E}-\chi_{S c h u ̈ E, E}^{\prime} \simeq\left(\chi_{E}-\chi_{S c h \ddot{u}}\right) \frac{\chi_{E}}{2 \chi_{S c h \ddot{u}}}\left(\alpha^{2}-\alpha^{\prime 2}\right) .
$$

Combining (41) and (46), one gets an approximate expression for $t_{S c h u ̈ E}^{\prime}-t_{S c h u ̈ E}$

$$
t_{S c h \ddot{u} E}^{\prime}-t_{S c h \ddot{u} E} \simeq a\left(t_{S c h \ddot{u} E}^{\prime}\right)\left(\chi_{E}-\chi_{S c h \ddot{u}}\right) \frac{\chi_{E}}{2 \chi_{S c h \ddot{u}}}\left(\alpha^{2}-\alpha^{\prime 2}\right),
$$

which may be used to deduce $t_{S c h \ddot{u} E}$ if $t_{S c h \ddot{u} E}^{\prime}$ has been determined from (37). Since $\alpha>\alpha^{\prime}$, the lower trajectory photon emerges from the Schücking sphere before the upper trajectory photon.

The upper trajectory photon emerges from the Schücking sphere with 4-velocity

$$
\dot{t}_{S c h \ddot{u} E}^{\prime}=\frac{1}{a_{S c h u ̈ E}^{\prime}}, \dot{\chi}_{S c h \ddot{u} E}^{\prime}=-\frac{\cos \left(\varphi_{S c h u ̈ E}^{\prime}-\alpha^{\prime}\right)}{a_{S c h \ddot{u} E}^{\prime 2}}, \dot{\varphi}_{S c h \ddot{u} E}^{\prime}=\frac{\chi_{p}^{\prime}}{a_{S c h u ̈ E}^{\prime 2} \chi_{S c h \ddot{u}}^{2}},
$$

where

$$
a_{S c h \ddot{u} E}^{\prime}:=a\left(t_{S c h \ddot{u} E}^{\prime}\right),
$$

and the lower trajectory photon emerges from the Schücking sphere with 4-velocity

$$
\dot{t}_{S c h \ddot{u} E}=\frac{1}{a_{S c h u ̈ E}}, \dot{\chi}_{S c h \ddot{u} E}=-\frac{\cos \left(\varphi_{S c h \ddot{u} E}+\alpha\right)}{a_{S c h \ddot{u} E}^{2}}, \dot{\varphi}_{S c h \ddot{u} E}=\frac{-\chi_{p}}{a_{S c h \ddot{u} E}^{2} \chi_{S c h \ddot{u}}^{2}},
$$

where

$$
a_{S c h u ̈ E}:=a\left(t_{S c h u ̈ E}\right) .
$$

Let $\gamma_{F}^{\prime}, \gamma_{F}$ be the smaller physical angles between the un-oriented direction of the upper trajectory photon and the direction towards the lens, and between the un-oriented direction of the lower trajectory photon and the direction towards the lens. We have

$$
\gamma_{F}^{\prime}=\arctan \left(\left|\chi_{S c h \ddot{u}} \frac{\dot{\varphi}_{S c h \ddot{u} E}^{\prime}}{\dot{\chi}_{S c h u ̈ E}^{\prime}}\right|\right)=\pi-\left(\varphi_{S c h \ddot{u} E}^{\prime}-\alpha^{\prime}\right)=\arcsin \left(\frac{\chi_{p}^{\prime}}{\chi_{S c h \ddot{u}}}\right)
$$

and

$$
\gamma_{F}=\arctan \left(\left|\chi_{S c h u ̈} \frac{\dot{\varphi}_{S c h \ddot{u} E}}{\dot{\chi}_{S c h u ̈ E}}\right|\right)=\pi+\left(\varphi_{S c h u ̈ E}+\alpha\right)=\arcsin \left(\frac{\chi_{p}}{\chi_{S c h \ddot{u}}}\right) .
$$


step 2: Determination of $t_{S c h u ̈ S}^{\prime}$ and $t_{S c h u ̈ S}$.

We have first to translate the 4-velocities of the upper and lower trajectories photons into the coordinates $(T, r, \varphi)$. They will serve as final conditions for the geodesic equation inside the Schücking sphere where prevails Kottler metric. Using the inverse Jacobian (13), one gets

$$
\dot{r}_{S c h \ddot{u} E}^{\prime}=\frac{C_{S c h u ̈ E}^{\prime}-\cos \left(\varphi_{S c h u ̈ E}^{\prime}-\alpha^{\prime}\right)}{a_{S c h u ̈ E}^{\prime}}
$$

and

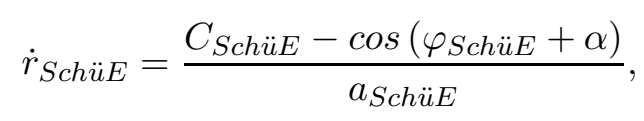

with

$$
C_{S c h u ̈ E}^{\prime}=C_{S c h \ddot{u}}\left(t_{S c h \ddot{u} E}^{\prime}\right)
$$

and

$$
C_{S c h \ddot{u} E}=C_{S c h \ddot{u}}\left(t_{S c h \ddot{u} E}\right),
$$

where $C_{S c h u ̈}(t)$ is given by

$$
\begin{aligned}
C_{S c h \ddot{u}}(t) & =\sqrt{1-B_{S c h \ddot{u}}(t)}=\sqrt{\frac{A}{a(t)} \chi_{S c h \ddot{u}}^{2}+\frac{\Lambda}{3} a^{2}(t) \chi_{S c h \ddot{u}}^{2}} \\
& =\chi_{S c h \ddot{u}} \sqrt{\frac{A}{a(t)}+\frac{\Lambda}{3} a^{2}(t) .}
\end{aligned}
$$

Let $\gamma_{K}^{\prime}$ and $\gamma_{K}$ denote respectively the smaller coordinate angles between the un-oriented direction of the upper trajectory photon and the direction towards the lens and between the un-oriented direction of the lower trajectory photon and the direction towards the lens. We have

$$
\gamma_{K}^{\prime}=: \arctan \left(\left|r_{S c h \ddot{u} E}^{\prime} \frac{\dot{\varphi}_{S c h \ddot{u} E}^{\prime}}{\dot{r}_{S c h \ddot{u} E}^{\prime}}\right|\right)=\arctan \left(\frac{\sin \gamma_{F}^{\prime}}{C_{S c h \ddot{u} E}^{\prime}+\cos \gamma_{F}^{\prime}}\right),
$$

and

$$
\gamma_{K}=: \arctan \left(\left|r_{S c h \ddot{u} E} \frac{\dot{\varphi}_{S c h u ̈ E}}{\dot{r}_{S c h \ddot{u} E}}\right|\right)=\arctan \left(\frac{\sin \gamma_{F}}{C_{S c h \ddot{u} E}+\cos \gamma_{F}}\right) .
$$

Moreover, we have at our disposal an initial condition, [12], which we may use to set $T_{\text {SchüE }}^{\prime}=t_{\text {Sch üE }}^{\prime}$. We have thus specified the final conditions of the geodesic equation inside the Schücking sphere.

Making use of the Christoffel symbols of the Kottler metric in the equatorial plane $\theta=\pi / 2$

$$
\Gamma_{T r}^{T}=\frac{B^{\prime}}{2 B}, \quad \Gamma_{T T}^{r}=\frac{B B^{\prime}}{2}, \quad \Gamma_{r r}^{r}=-\frac{B^{\prime}}{2 B}, \quad \Gamma_{\varphi \varphi}^{r}=-r B, \quad \Gamma_{r \varphi}^{\varphi}=\frac{1}{r},
$$

the geodesic equations then read

$$
\begin{aligned}
\ddot{T}+\frac{B^{\prime}(r)}{B(r)} \dot{T} \dot{r} & =0, \\
\ddot{r}+\frac{1}{2} B(r) B^{\prime}(r) \dot{T}^{2}-\frac{1}{2} \frac{B^{\prime}(r)}{B(r)} \dot{r}^{2}-r B(r) \dot{\varphi}^{2} & =0, \\
\ddot{\varphi}+\frac{2}{r} \dot{r} \dot{\varphi} & =0,
\end{aligned}
$$


from which we deduce three first integrals

$$
\begin{array}{r}
\dot{T}=1 / B(r), \\
\dot{\varphi} r^{2}=J, \\
\frac{\dot{r}^{2}}{B(r)}+\frac{J^{2}}{r^{2}}-\frac{1}{B(r)}=-E
\end{array}
$$

Equation (67) comes from invariance of the metric under rotations and $J$ has the meaning of an angular momentum per unit mass, (68) comes from invariance of the metric under time translations and $E$ has the meaning of energy per unit mass. For the photon $E=0$. Eliminating the affine parameter between (67) and (68), one gets in the case of a photon

$$
\frac{d r}{d \varphi}= \pm r \sqrt{\frac{r^{2}}{J^{2}}-B}
$$

At the peri-lens $r_{p}^{\prime}$ for the upper trajectory photon $\left.\frac{d r}{d \varphi}\right|_{r_{p}^{\prime}}=0$, from which one deduces the expression of $J$ in terms of $r_{p}^{\prime}$

$$
J=\frac{r_{p}^{\prime}}{\sqrt{B\left(r_{p}^{\prime}\right)}}
$$

Similarly at the peri-lens $r_{p}$ for the lower trajectory photon $\left.\frac{d r}{d \varphi}\right|_{r_{p}}=0$ and one deduces another expression for $J$ in terms of $r_{p}$

$$
J=\frac{r_{p}}{\sqrt{B\left(r_{p}\right)}} .
$$

Replacing $J$ by the appropriate expression (70) or (71), one gets

$$
\frac{d \varphi}{d r}= \pm \frac{1}{r \sqrt{r^{2} / r_{p}^{\prime 2}-1}}\left(1-\frac{s}{r}-\frac{s}{r_{p}^{\prime}} \frac{r}{r+r_{p}^{\prime}}\right)^{-1 / 2}
$$

valid for the upper trajectory photon and

$$
\frac{d \varphi}{d r}= \pm \frac{1}{r \sqrt{r^{2} / r_{p}^{2}-1}}\left(1-\frac{s}{r}-\frac{s}{r_{p}} \frac{r}{r+r_{p}}\right)^{-1 / 2} .
$$

valid for the lower trajectory photon, where $s$ denotes the Schwarzschild radius $s=2 G M$. It is worthwhile to notice that the cosmological constant has disappeared from (72) and also from (73).

Since $s / r_{p}^{\prime} \ll 1$ and $s / r_{p} \ll 1$, we will hereafter only retain terms up to linear order in $s / r_{p}^{\prime}$ or in $s / r_{p}$. In this approximation

$$
r_{p}^{\prime} \simeq a\left(t_{S c h \ddot{u} E}^{\prime}\right) \chi_{S c h \ddot{u}} \sin \gamma_{K}^{\prime}-G M
$$

and

$$
r_{p} \simeq a\left(t_{S c h \ddot{u} E}\right) \chi_{S c h \ddot{u}} \sin \gamma_{K}-G M .
$$


Eliminating now the affine parameter between (66) and (68) and taking into account (70), one gets

$$
\frac{d T}{d r}= \pm \frac{1}{B(r) \sqrt{1-\frac{r_{p}^{\prime 2}}{r^{2}} \frac{B(r)}{B\left(r_{p}^{\prime}\right)}}}
$$

for the upper trajectory photon and

$$
\frac{d T}{d r}= \pm \frac{1}{B(r) \sqrt{1-\frac{r_{p}^{2}}{r^{2}} \frac{B(r)}{B\left(r_{p}\right)}}}
$$

for the lower trajectory photon.

Let us denote $T_{S c h u ̈ S}^{\prime}$ and $T_{S c h \ddot{u} E}^{\prime}$ the Kottler times at which the upper trajectory photon penetrates inside and emerges from the vacuole respectively and $t_{S c h \ddot{u} S}^{\prime}$ and $t_{S c h \ddot{u} E}^{\prime}$ the corresponding Friedmann times. From now on, we will denote the expression of $d \varphi / d r$ and $d T / d r$ valid for the upper trajectory photon by $d \varphi^{\prime} / d r$ and $d T^{\prime} / d r$ respectively and will continu to denote the expressions valid for the lower trajectory by $d \varphi / d r$ and $d T / d r$. We have

$$
T_{S c h \ddot{u} E}^{\prime}-T_{S c h \ddot{u} S}^{\prime}=\int_{r_{P}^{\prime}}^{r\left(t_{S c h \ddot{u} E}^{\prime}\right)}\left|\frac{d T^{\prime}}{d r}\right| d r+\int_{r_{p}^{\prime}}^{r\left(t_{S c h u ̈ S}^{\prime}\right)}\left|\frac{d T^{\prime}}{d r}\right| d r,
$$

with $r(t)=a(t) \chi_{S c h u ̈}$ and where $d T^{\prime} / d r$ is given by (76). To obtain (78), we have used the fact that $T^{\prime}$ decreases when $r$ increases from $r\left(t_{S c h u ̈ S}^{\prime}\right)$ to $r_{p}^{\prime}$ as well as when $r$ increases from $r_{p}^{\prime}$ to $r\left(t_{S c h u ̈ E}^{\prime}\right)$.

Using the relation (14) relating the Kottler time $T$ and the Friedmann time $t$, one gets

$$
T_{S c h \ddot{u} S}^{\prime}=T_{S c h \ddot{u} E}^{\prime}-\int_{t_{S c h u ̈ S}^{\prime}}^{t_{S c h u ̈ E}^{\prime}} \frac{d t}{B_{S c h u ̈}(t)} .
$$

Moreover, we have at our disposal a free initial condition [12] which we may use to set $t_{\text {SchüE }}^{\prime}=T_{S c h \ddot{u} E}^{\prime}$ for instance. Combining (78) and (79), one gets

$$
\int_{r_{P}^{\prime}}^{r\left(t_{\text {SchüE }}^{\prime}\right)}\left|\frac{d T^{\prime}}{d r}\right| d r+\int_{r_{P}^{\prime}}^{r\left(t_{\text {SchüS }}^{\prime}\right)}\left|\frac{d T^{\prime}}{d r}\right| d r-\int_{t_{\text {SchüS }}^{\prime}}^{t_{\text {SchüE }}^{\prime}} \frac{d t}{B_{\text {Schü }}(t)}=0,
$$

from which one can deduce $t_{S c h u ̈ S}^{\prime}$. If one is interested in $T_{S c h u ̈ S}^{\prime}$ one can use (79) to obtain it.

Let us denote $T_{S c h u ̈ S}$ and $T_{S c h u ̈ E}$ the Kottler times at which the lower trajectory photon penetrates into and leaves the Schücking sphere respectively and by $t_{S c h u ̈ S}$ and $t_{S c h u ̈ E}$ the corresponding Friedmann times.

To compute $t_{S c h u ̈ S}$, one can proceed in a similar manner as for $t_{S c h u ̈ S}^{\prime}$. We have the analogous of (78), (79) and (80)

$$
\begin{aligned}
& T_{S c h \ddot{u} E}-T_{S c h \ddot{u} S}=\int_{r_{P}}^{r\left(t_{S c h u ̈ E}\right)}\left|\frac{d T}{d r}\right| d r+\int_{r_{P}^{\prime}}^{r\left(t_{S c h u ̈ S}\right)}\left|\frac{d T}{d r}\right| d r, \\
& T_{S c h \ddot{u} S}=T_{S c h \ddot{u} E}-\int_{t_{S c h u ̈ S}}^{t_{S c h u ̈ E}} \frac{d t}{B_{S c h u ̈}(t)}
\end{aligned}
$$


and

$$
\int_{r_{P}}^{r\left(t_{S c h u ̈ E}\right)}\left|\frac{d T}{d r}\right| d r+\int_{r_{P}}^{r\left(t_{S c h u ̈ S}\right)}\left|\frac{d T}{d r}\right| d r-\int_{t_{S c h u ̈ S}}^{t_{S c h u ̈ E}} \frac{d t}{B_{S c h u ̈}(t)}=0,
$$

from which one can deduce $t_{S c h u ̈ S}$.

But as in the cas of $t_{S c h u ̈ E}$, we prefer to proceed in a different manner: determining $t_{S c h u ̈}$ by differences. Combining (78) and (81), one obtains

$$
\begin{aligned}
& \left(T_{S c h \ddot{u} E}^{\prime}-T_{S c h \ddot{u} S}^{\prime}\right)-\left(T_{S c h u ̈ E}-T_{S c h \ddot{u} S}\right)=\int_{r_{p}^{\prime}}^{r\left(t_{\text {SchüE }}^{\prime}\right)}\left|\frac{d T^{\prime}}{d r}\right| d r \\
& +\int_{r_{p}^{\prime}}^{r\left(t_{S c h u ̈ S}^{\prime}\right)}\left|\frac{d T^{\prime}}{d r}\right| d r-\int_{r_{p}}^{r\left(t_{S c h u ̈ E}\right)}\left|\frac{d T}{d r}\right| d r-\int_{r_{p}}^{r\left(t_{S c h u ̈ S}\right)}\left|\frac{d T}{d r}\right| d r .
\end{aligned}
$$

On the other hand, using (14), we have

$$
T_{S c h \ddot{u} E}^{\prime}-T_{S c h \ddot{u} E}=\int_{t_{S c h \ddot{u} E}}^{t_{S c h \ddot{u} E}^{\prime}} \frac{d t}{B_{S c h \ddot{u}}(t)} \sim \frac{t_{S c h \ddot{u} E}^{\prime}-t_{S c h \ddot{u} E}}{B_{S c h \ddot{u}}\left(t_{S c h u ̈ E}^{\prime}\right)},
$$

and

$$
T_{S c h \ddot{u} S}^{\prime}-T_{S c h \ddot{u} S}=\int_{t_{S c h u ̈ S}}^{t_{S c h \ddot{u} S}^{\prime}} \frac{d t}{B_{S c h \ddot{u}}(t)} \sim \frac{t_{S c h \ddot{u} S}^{\prime}-t_{S c h \ddot{u} S}}{B_{S c h \ddot{u}}\left(t_{S c h u ̈ S}^{\prime}\right)},
$$

where we have used the fact that $B_{S c h u ̈}$ does not vary appreciably on the time intervals $\left[t_{S c h \ddot{u} E}, t_{S c h \ddot{u} E}^{\prime}\right]$ and $\left[t_{S c h u ̈ S}, t_{S c h \ddot{u} S}^{\prime}\right]$ since these are smaller than cosmological scales.

Substituting (85) and (86) into (84) one gets

$$
\begin{aligned}
& \frac{t_{S c h \ddot{u} E}^{\prime}-t_{S \operatorname{shu} E}}{B_{S c h \ddot{u}}\left(t_{S c h \ddot{u} E}^{\prime}\right)}-\frac{t_{S \operatorname{sch} S}^{\prime}-t_{S \operatorname{sch} \ddot{u} S}}{B_{S c h \ddot{u}}\left(t_{S \operatorname{sch} u}^{\prime}\right)} \simeq \\
& \int_{r_{p}^{\prime}}^{r\left(t_{S c h \ddot{u} E}^{\prime}\right)}\left|\frac{d T^{\prime}}{d r}\right| d r+\int_{r_{p}^{\prime}}^{r\left(t_{S c h \ddot{u} S}^{\prime}\right)}\left|\frac{d T^{\prime}}{d r}\right| d r \\
& -\int_{r_{p}}^{r\left(t_{S c h u ̈ E}\right)}\left|\frac{d T}{d r}\right| d r-\int_{r_{p}}^{r\left(t_{S c h u ̈ S}\right)}\left|\frac{d T}{d r}\right| d r \\
& \simeq \quad \int_{r_{p}^{\prime}}^{r\left(t_{S c h \ddot{u} E}^{\prime}\right)}\left|\frac{d T^{\prime}}{d r}\right| d r-\int_{r_{p}}^{r\left(t_{S c h \ddot{u} E}^{\prime}\right)}\left|\frac{d T}{d r}\right| d r \\
& +\int_{r_{p}^{\prime}}^{r\left(t_{S c h u ̈ S}^{\prime}\right)}\left|\frac{d T^{\prime}}{d r}\right| d r-\int_{r_{p}}^{r\left(t_{S c h u ̈ S}^{\prime}\right)}\left|\frac{d T}{d r}\right| d r \\
& -\int_{r\left(t_{\text {SchüE }}^{\prime}\right)}^{r\left(t_{\text {SchüE }}\right)}\left|\frac{d T}{d r}\right| d r-\int_{r\left(t_{\text {SchüS }}^{\prime}\right)}^{r\left(t_{\text {SchüS }}\right)}\left|\frac{d T}{d r}\right| d r .
\end{aligned}
$$

But since we deal with smaller length and time scales than cosmological ones:

$$
\begin{aligned}
& \int_{r\left(t_{\text {SchüE }}^{\prime}\right)}^{r\left(t_{\text {SchüE }}\right)}\left|\frac{d T}{d r}\right| d r \simeq\left|\frac{d T}{d r}\right|_{r\left(t_{\text {SchüE }}^{\prime}\right)}\left[a\left(t_{\text {SchüE }}\right)-a\left(t_{\text {SchüE }}^{\prime}\right)\right] \chi_{S c h \ddot{u}}, \\
& \int_{r\left(t_{\text {SchüS }}^{\prime}\right)}^{r\left(t_{\text {SchüS }}\right)}\left|\frac{d T}{d r}\right| d r \simeq\left|\frac{d T}{d r}\right|_{r\left(t_{\text {SchüS }}^{\prime}\right)}\left[a\left(t_{\text {SchüS }}\right)-a\left(t_{\text {SchüS }}^{\prime}\right)\right] \chi_{S c h \ddot{u} .}
\end{aligned}
$$


Using equation (6), one deduces

$$
\begin{aligned}
& a\left(t_{\text {SchüE }}\right)-a\left(t_{\text {SchüE }}^{\prime}\right) \simeq \sqrt{\frac{A}{a\left(t_{\text {SchüE }}^{\prime}\right)}+\frac{\Lambda}{3} a^{2}\left(t_{\text {Sch } \ddot{E} E}^{\prime}\right)}\left(t_{\text {SchüE}}-t_{\text {SchüE }}^{\prime}\right), \\
& a\left(t_{S c h \ddot{u} S}\right)-a\left(t_{S c h \ddot{u} S}^{\prime}\right) \simeq \sqrt{\frac{A}{a\left(t_{S c h \ddot{u} S}^{\prime}\right)}+\frac{\Lambda}{3} a^{2}\left(t_{S c h \ddot{u} S}^{\prime}\right)}\left(t_{S c h \ddot{u} S}-t_{S c h \ddot{u} S}^{\prime}\right),
\end{aligned}
$$

with

$$
A=\frac{1}{3} \rho_{\text {dust }, 0} a_{0}^{3}=\frac{1}{3} \rho_{\text {dust }, 0} .
$$

On the other hand, using the results of reference [12]

$$
\begin{aligned}
& \int_{r_{p}^{\prime}}^{r\left(t_{S c h \ddot{u} E}^{\prime}\right)}\left|\frac{d T^{\prime}}{d r}\right| d r-\int_{r_{p}}^{r\left(t_{S c h \ddot{u} E}^{\prime}\right)}\left|\frac{d T}{d r}\right| \simeq \frac{M}{8 \pi}\left[\frac{1}{2}\left(1-\frac{r_{p}^{\prime 2}}{r_{p}^{2}}\right) \frac{8 \pi r_{p}^{2}}{M r\left(t_{S c h \ddot{u} E}^{\prime}\right)}\right. \\
& \left.-\frac{3}{2}\left(\frac{r_{p}^{2}}{r_{p}^{\prime 2}}-1\right) \frac{M}{8 \pi r_{p}^{2} \sqrt{\Lambda / 3}} \operatorname{arctanh}\left(\sqrt{\frac{\Lambda}{3}} r\left(t_{S c h u ̈ E}^{\prime}\right)\right)-2 \ln \left(\frac{r_{p}^{\prime}}{r_{p}}\right)\right] .
\end{aligned}
$$

It suffices to make the following replacements

$$
x \rightarrow \frac{r_{p}^{\prime}}{r_{p}}, \epsilon_{T} \rightarrow \frac{r_{p}}{r\left(t_{\text {SchüE }}^{\prime}\right)}, \delta \rightarrow \frac{M}{8 \pi r_{p}}, \lambda \rightarrow r_{p} \sqrt{\Lambda / 3}
$$

in equation (23) of reference [17].

In the same manner

$$
\begin{aligned}
& \int_{r_{p}^{\prime}}^{r\left(t_{\text {SchüS }}^{\prime}\right)}\left|\frac{d T^{\prime}}{d r}\right| d r-\int_{r_{p}}^{r\left(t_{\text {SchüS }}^{\prime}\right)}\left|\frac{d T}{d r}\right| \simeq \frac{M}{8 \pi}\left[\frac{1}{2}\left(1-\frac{r_{p}^{\prime 2}}{r_{p}^{2}}\right) \frac{8 \pi r_{p}^{2}}{M r\left(t_{S c h \ddot{u} S}^{\prime}\right)}\right. \\
& \left.-\frac{3}{2}\left(\frac{r_{p}^{2}}{r_{p}^{\prime 2}}-1\right) \frac{M}{8 \pi r_{p}^{2} \sqrt{\Lambda / 3}} \operatorname{arctanh}\left(\sqrt{\frac{\Lambda}{3}} r\left(t_{S c h \ddot{u} S}^{\prime}\right)\right)-2 \ln \left(\frac{r_{p}^{\prime}}{r_{p}}\right)\right] .
\end{aligned}
$$

Collecting together the previous results, one gets an analytical approximation for $t_{S c h u ̈ S}-$ $t_{\text {SchüS }}^{\prime}$

$$
\begin{aligned}
& t_{S c h \ddot{u} S}-t_{S c h \ddot{u} S}^{\prime} \simeq\left\{\frac { M } { 8 \pi } \left[\frac{1}{2}\left(1-\frac{r_{p}^{\prime 2}}{r_{p}^{2}}\right) \frac{8 \pi r_{p}^{2}}{M_{\text {Schü }}}\left(\frac{1}{a\left(t_{\text {SchüE }}^{\prime}\right)}+\frac{1}{a\left(t_{\text {Schü } S}^{\prime}\right)}\right)\right.\right.
\end{aligned}
$$

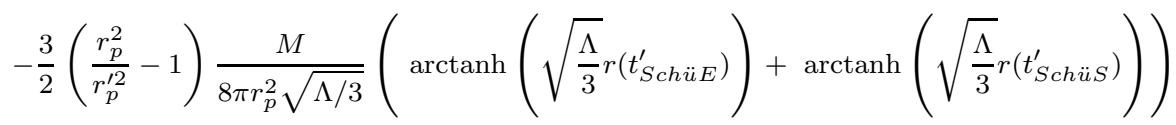

$$
\begin{aligned}
& \left.-4 \ln \left(\frac{r_{p}^{\prime}}{r_{p}}\right)\right] \\
& +\left[\chi_{S c h \ddot{u}} \sqrt{\frac{A}{a\left(t_{S c h u ̈ E}^{\prime}\right)}+\frac{\Lambda}{3} a^{2}\left(t_{S c h u ̈ E}^{\prime}\right)}\left|\frac{d T}{d r}\right|_{r\left(t_{S c h \ddot{u} E}^{\prime}\right)}-\frac{1}{B_{S c h \ddot{u}}\left(t_{S c h \ddot{u} E}^{\prime}\right)}\right] \\
& \left.\times a\left(t_{S c h \ddot{u} E}^{\prime}\right)\left(\chi_{E}-\chi_{S c h \ddot{u}}\right) \frac{\chi_{E}}{2 \chi_{S c h \ddot{u}}}\left(\alpha^{2}-\alpha^{\prime 2}\right)\right\} \\
& \times\left(\frac{1}{B_{S c h \ddot{u}}\left(t_{\text {Schü } S}^{\prime}\right)}+\chi_{\text {Sch } \ddot{u}} \sqrt{\frac{A}{a\left(t_{\text {Sch } u}^{\prime}\right)}+\frac{\Lambda}{3} a^{2}\left(t_{\text {SchüS }}^{\prime}\right)}\left|\frac{d T}{d r}\right|_{r\left(t_{\text {Schü } S}^{\prime}\right)}\right)^{-1},
\end{aligned}
$$


where we have also used the expression (47) for $\left(t_{S c h \ddot{u} E}^{\prime}-t_{S c h \ddot{u} E}\right)$. Then the knowledge of $t_{S c h u ̈ S}^{\prime}$ allows one to deduce $t_{S c h \ddot{I} S}$.

Let us now determine in turn the polar angles $\varphi_{\text {SchüS }}^{\prime}$ and $\varphi_{\text {SchüS }}$ at which the upper and lower trajectories photons penetrate inside the Schücking sphere. Since the angle $\varphi^{\prime}$ increases all the way from $r_{S c h u ̈ S}^{\prime}$ to $r_{p}^{\prime}$ and from $r_{p}^{\prime}$ to $r_{S c h u ̈ E}^{\prime}$, one gets

$$
\varphi_{S c h \ddot{u} S}^{\prime}=\varphi_{S c h \ddot{u} E}^{\prime}-\int_{r_{p}^{\prime}}^{r\left(t_{S c h \ddot{u} S}^{\prime}\right)}\left|\frac{d \varphi^{\prime}}{d r}\right| d r-\int_{r_{p}^{\prime}}^{r\left(t_{S c h \ddot{u} E}^{\prime}\right)}\left|\frac{d \varphi^{\prime}}{d r}\right| d r .
$$

To the linear order in the ratio $s / r_{p}^{\prime}$, Schwarzschild radius $s=2 G M$ divided by peri-lens $r_{p}^{\prime}$, one gets [12]

$$
\begin{gathered}
\varphi_{\text {SchüS } \sim}^{\prime} \varphi_{S c h \ddot{u} E}^{\prime}-\pi+\arcsin \left(\frac{r_{p}^{\prime}}{r_{S c h \ddot{u} E}^{\prime}}\right)+\arcsin \left(\frac{r_{p}^{\prime}}{r_{S c h \ddot{u} S}^{\prime}}\right) \\
-\frac{1}{2} \frac{s}{r_{S c h \ddot{u} E}^{\prime}} \sqrt{\frac{r_{S c h u ̈ E}^{\prime 2}}{r_{p}^{\prime 2}}-1}-\frac{1}{2} \frac{s}{r_{S c h \ddot{u} S}^{\prime}} \sqrt{\frac{r_{S c h \ddot{u} S}^{\prime 2}}{r_{p}^{\prime 2}}-1} \\
-\frac{1}{2} \frac{s}{r_{p}^{\prime}} \sqrt{\frac{r_{S c h \ddot{u} E}^{\prime}-r_{p}^{\prime}}{r_{S c h \ddot{u} E}^{\prime}+r_{p}^{\prime}}}-\frac{1}{2} \frac{s}{r_{p}^{\prime}} \sqrt{\frac{r_{S c h \ddot{u} S}^{\prime}-r_{p}^{\prime}}{r_{S c h \ddot{u} S}^{\prime}+r_{p}^{\prime}}} .
\end{gathered}
$$

Let us now compute the polar angle $\varphi_{S c h u ̈ S}$ at which the lower trajectory photon penetrates inside the Schücking sphere. Since $\varphi$ decreases when $r$ varies from $r_{S c h u ̈ S}$ to $r_{p}$ and also when $r$ varies from $r_{p}$ to $r_{S c h u ̈ E}$, then

$$
\varphi_{S c h \ddot{u} S}=\varphi_{S c h \ddot{u} E}+\int_{r_{p}}^{r\left(t_{S c h u ̈ S}\right)}\left|\frac{d \varphi}{d r}\right| d r+\int_{r_{p}}^{r\left(t_{S c h u ̈ E}\right)}\left|\frac{d \varphi}{d r}\right| d r .
$$

To the linear order in the ratio $s / r_{p}$, one gets for $\varphi_{S c h u ̈ S}[12$

$$
\begin{aligned}
& \varphi_{S c h \ddot{u} S} \simeq \varphi_{S c h \ddot{u} E}+\pi-\arcsin \left(\frac{r_{P}}{r_{S c h \ddot{u} E}}\right)-\arcsin \left(\frac{r_{P}}{r_{S c h \ddot{u} S}}\right) \\
& +\frac{M}{8 \pi} \frac{1}{r_{S c h u ̈ E}} \sqrt{\frac{r_{S c h \ddot{u} E}^{2}}{r_{p}^{2}}-1}+\frac{M}{8 \pi} \frac{1}{r_{S c h u ̈ S}} \sqrt{\frac{r_{S c h \ddot{u} S}^{2}}{r_{p}^{2}}-1} \\
& +\frac{M}{8 \pi} \frac{1}{r_{p}} \sqrt{\frac{r_{S c h \ddot{u} E}-r_{p}}{r_{S c h u ̈ E}+r_{p}}}+\frac{M}{8 \pi} \frac{1}{r_{p}} \sqrt{\frac{r_{S c h \ddot{u} S}-r_{p}}{r_{S c h \ddot{u} S}+r_{p}}} .
\end{aligned}
$$

step 3: Determination of $t_{S}^{\prime}$ and $t_{S}$

One can now compute $\varphi_{S}^{\prime}$

$$
\varphi_{S}^{\prime}=\varphi_{S c h \ddot{u} S}^{\prime}-\gamma_{F S}^{\prime}+\arcsin \left(\frac{\chi_{S c h \ddot{u}}}{\chi_{L, S}} \sin \gamma_{F S}^{\prime}\right),
$$

where $\varphi_{S c h \ddot{u} S}^{\prime}$ is given by (97) and where $\gamma_{F S}^{\prime}$ the smaller physical angle between the unoriented direction of the photon and the direction towards the lens as the photon penetrates inside the Schücking sphere from the external side:

$$
\gamma_{F S}^{\prime}=\arctan \left(\left|\chi_{S c h \ddot{u}} \frac{\dot{\varphi}_{S c h u ̈ S}^{\prime}}{\dot{\chi}_{S c h u ̈ S}^{\prime}}\right|\right)=\arctan \left(-\chi_{S c h \ddot{u}} \frac{\dot{\varphi}_{S c h u ̈ S}^{\prime}}{\dot{\chi}_{S c h u ̈ S}^{\prime}}\right),
$$


where $\dot{\varphi}_{S c h \ddot{u} S}^{\prime}$ and $\dot{\chi}_{S c h \ddot{u} S}^{\prime}$ are given respectively by

$$
\dot{\varphi}_{S c h u ̈ S}^{\prime}=\frac{r_{p}^{\prime}}{r_{S c h u ̈ S}^{\prime 2} \sqrt{B\left(r_{p}^{\prime}\right)}}=\frac{r_{p}^{\prime}}{a^{2}\left(t_{S c h \ddot{u} S}^{\prime}\right) \chi_{S c h \ddot{u}}^{2} \sqrt{B\left(r_{p}^{\prime}\right)}},
$$

and

$$
\begin{aligned}
& \dot{\chi}_{S c h \ddot{u} S}^{\prime}=-\frac{1}{a\left(t_{S c h \ddot{u} S}^{\prime}\right) B_{S c h \ddot{u}}\left(t_{S c h \ddot{u} S}^{\prime}\right)} \sqrt{1-\frac{r_{p}^{\prime 2}}{a^{2}\left(t_{S c h u ̈ S}^{\prime}\right) \chi_{S c h \ddot{u}}^{2}} \frac{B_{S c h \ddot{u}}\left(t_{S c h \ddot{u} S}^{\prime}\right)}{B\left(r_{p}^{\prime}\right)}} \\
& -\frac{C_{S c h \ddot{u}}\left(t_{S c h \ddot{u} S}^{\prime}\right)}{a\left(t_{S c h \ddot{u} S}^{\prime}\right)} \frac{1}{B_{S c h \ddot{u}}\left(t_{S c h \ddot{u} S}^{\prime}\right)},
\end{aligned}
$$

with

$$
C_{S c h \ddot{u}}\left(t_{S c h \ddot{u} S}^{\prime}\right)=\sqrt{\frac{A}{a\left(t_{S c h \ddot{u} S}^{\prime}\right)} \chi_{S c h \ddot{u}}^{2}+\frac{\Lambda}{3} a^{2}\left(t_{S c h \ddot{u} S}^{\prime}\right) \chi_{S c h \ddot{u}}^{2}}
$$

and

$$
B_{S c h \ddot{u}}\left(t_{S c h \ddot{u} S}^{\prime}\right)=1-\frac{A}{a\left(t_{S c h u ̈ S}^{\prime}\right)} \chi_{S c h \ddot{u}}^{2}-\frac{\Lambda}{3} a^{2}\left(t_{S c h \ddot{u} S}^{\prime}\right) \chi_{S c h \ddot{u}}^{2} .
$$

To obtain (103) we have used the Jacobian of the coordinate transformation $(T, r) \rightarrow(t, \chi)$, (13), together with the expressions of $\dot{T}_{S c h u ̈ S}^{\prime}$ and $\dot{r}_{S c h \ddot{u} S}^{\prime}$

$$
\dot{T}_{\text {SchüS }}^{\prime}=\frac{1}{B\left(t_{\text {SchüS }}^{\prime}\right)}, \quad \dot{r}_{\text {SchüS }}^{\prime}=-\sqrt{1-\frac{r_{p}^{\prime 2}}{r_{\text {SchüS }}^{\prime 2}} \frac{B\left(t_{\text {SchüS }}^{\prime}\right)}{B\left(r_{p}^{\prime}\right)}} .
$$

$\chi_{L, S}$ is the geodesic distance between the source and the lens, which can be accurately approximated by

$$
\chi_{L, S} \simeq \chi_{S}-\chi_{L}
$$

In the same manner, once $\varphi_{S c h u ̈ S}$ determined, one can compute the polar angle $\varphi_{S}$ corresponding to the source by a relation analogous to (100)

$$
\varphi_{S}=\varphi_{S c h \ddot{u} S}+\gamma_{F S}-\arcsin \left(\frac{\chi_{S c h \ddot{u}}}{\chi_{L, S}} \sin \gamma_{F S}\right),
$$

where $\varphi_{S c h \ddot{i} S}$ is given by (99) and where $\gamma_{F S}$ is the smaller physical angle between the un-oriented direction of the lower trajectory photon and the direction towards the lens as the photon penetrates inside the Schücking sphere from the external side:

$$
\gamma_{F S}=\arctan \left(\left|\chi_{S c h u ̈} \frac{\dot{\varphi}_{S c h u ̈ S}}{\dot{\chi}_{S c h u ̈ S}}\right|\right)=\arctan \left(\chi_{S c h u ̈} \frac{\dot{\varphi}_{S c h u ̈ S}}{\dot{\chi}_{S c h u ̈ S}}\right) .
$$

Using

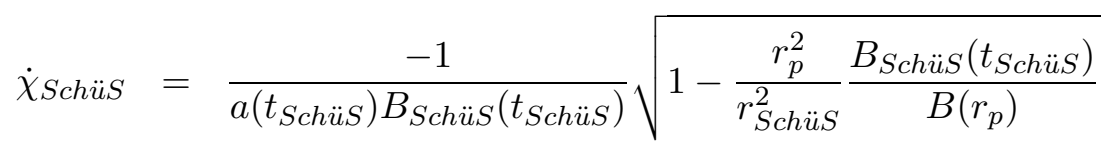

$$
\begin{aligned}
& -\frac{C_{S c h \ddot{u} S}\left(t_{S c h \ddot{u} S}\right)}{a\left(t_{S c h u ̈ S}\right) B_{S c h \ddot{u} S}\left(t_{S c h u ̈ S}\right)},
\end{aligned}
$$


obtained by using the Jacobian of the coordinate transformation $(T, r) \rightarrow(t, \chi)$ and

$$
\dot{\varphi}_{S c h u ̈ S}=-\frac{r_{p}}{a^{2}\left(t_{S c h u ̈ S}\right) \chi_{S c h u ̈}^{2} \sqrt{B\left(r_{p}\right)}},
$$

then

$$
\gamma_{F S}=\arctan \left(\frac{r_{p} B_{S c h u ̈ S}\left(t_{S c h u ̈ S}\right)}{r_{S c h u ̈ S} \sqrt{B\left(r_{p}\right)}\left(C_{S c h u ̈ S}\left(t_{S c h u ̈ S}\right)+\sqrt{1-\frac{r_{p}^{2}}{r_{S c h u ̈ S}^{2}} \frac{B_{S c h u ̈ S}\left(t_{S c h u ̈ S}\right)}{B\left(r_{p}\right)}}\right)}\right) .
$$

For a given $M$, one obtains in turn $t_{S c h \ddot{u} E}^{\prime}, t_{S c h \ddot{u} S}^{\prime}, \varphi_{S c h u ̈ S}^{\prime}, \varphi_{S}^{\prime}, t_{S c h \ddot{u} E}, t_{S c h u ̈ S}, \varphi_{S c h u ̈ S}$ and $\varphi_{S}$.

In general $\varphi_{S}^{\prime} \neq \varphi_{S}$. To achieve $\varphi_{S}^{\prime}=\varphi_{S}$, which corresponds to the fact that the upper and the lower trajectories photons are emitted by the same source, we have to adjust $M$, i.e., we have to vary $M$ until the equality $\varphi_{S}^{\prime}=\varphi_{S}$ is satisfied. We end up with values of $M, t_{S c h \ddot{u} E}^{\prime}, t_{S c h \ddot{u} S}^{\prime}, \varphi_{S c h \ddot{u} S}^{\prime}, t_{S c h \ddot{u} E}, t_{S c h u ̈ S}, \varphi_{S c h \ddot{I} S}$ and $\varphi_{S}^{\prime}=\varphi_{S}$.

We are now in a position to determine $t_{S}-t_{S}^{\prime}$.

Using once again some elementary Euclidean geometry, similar to that used to obtain $\chi_{S c h \ddot{u} E, E(30)}^{\prime}$, one obtains for the geodesic distance $\chi_{S c h u ̈ S, S}^{\prime}$ between the source $S$ and the photon of the upper trajectory as it crosses the Schücking sphere in the half space containing the source:

$$
\chi_{S, S c h \ddot{u} S}^{\prime}=\sqrt{\chi_{L, S}^{2}+\chi_{S c h \ddot{u}}^{2}-2 \chi_{L, S} \chi_{S c h \ddot{u}} \cos \left(\varphi_{S c h \ddot{u} S}^{\prime}-\varphi_{S}\right)} .
$$

Proceeding in the same manner, we get for the geodesic distance $\chi_{S c h u ̈ S, S}$ between the source $S$ and the photon of the lower trajectory as it crosses the Schücking sphere in the half space containing the source

$$
\chi_{S c h \ddot{u} S, S}=\sqrt{\chi_{L, S}^{2}+\chi_{S c h \ddot{u}}^{2}-2 \chi_{L, S} \chi_{S c h \ddot{u}} \cos \left(\varphi_{S c h \ddot{u} S}-\varphi_{S}\right)} .
$$

Making use of the approximations

$$
\cos x \simeq 1-x^{2} / 2 \quad \text { and } \quad \sqrt{1+x} \simeq 1+x / 2,
$$

valid for $|x| \ll 1$, one gets

$$
\begin{aligned}
\chi_{S c h \ddot{u} S, S}^{\prime} & \simeq\left(\chi_{L, S}-\chi_{S c h \ddot{u}}\right)-\frac{\left(\varphi_{S \operatorname{schu} S}^{\prime}-\varphi_{S}\right)^{2}}{2\left(\chi_{L, S}^{-1}-\chi_{S c h \ddot{u}}^{-1}\right)}, \\
\chi_{S c h \ddot{u} S, S} & \simeq\left(\chi_{L, S}-\chi_{S c h \ddot{u}}\right)-\frac{\left(\varphi_{S c h \ddot{u} S}-\varphi_{S}\right)^{2}}{2\left(\chi_{L, S}^{-1}-\chi_{S c h u ̈}^{-1}\right)} .
\end{aligned}
$$

Then

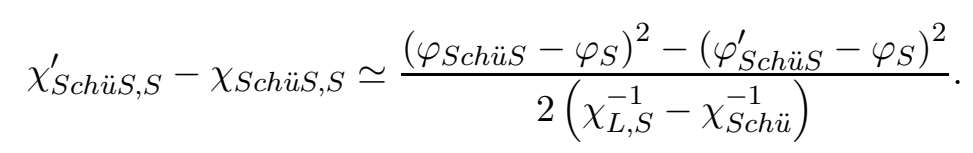


On the other hand, equation (18) with the final condition $\chi\left(t_{S c h \ddot{u} S}^{\prime}\right)=0$ gives

$$
0-\chi_{S c h u ̈ S, S}^{\prime}=-\int_{t_{S}^{\prime}}^{t_{S c h u ̈ S}^{\prime}} \frac{d t}{a(t)}
$$

In a similar manner, from (18) with the final condition $\chi\left(t_{\text {SchüS }}\right)=0$, one gets

$$
0-\chi_{S c h u ̈ S, S}=-\int_{t_{S}}^{t_{S c h u ̈ S}} \frac{d t}{a(t)} .
$$

From (119) and (120), one deduces an expression for $\chi_{S c h u ̈ S, S}-\chi_{S c h u ̈ S, S}^{\prime}$

$$
\begin{aligned}
\chi_{S c h \ddot{u} S, S}-\chi_{S \operatorname{schu} S, S}^{\prime} & =\int_{t_{S}}^{t_{S c h u ̈}} \frac{d t}{a(t)}-\int_{t_{S}^{\prime}}^{t_{S c h u ̈ S}^{\prime}} \frac{d t}{a(t)} \\
& =\int_{t_{S}}^{t_{S}^{\prime}} \frac{d t}{a(t)}+\int_{t_{S}^{\prime}}^{t_{S c h u ̈ S}} \frac{d t}{a(t)}-\int_{t_{S}^{\prime}}^{t_{S c h u ̈ S}^{\prime}} \frac{d t}{a(t)} \\
& =\int_{t_{S}}^{t_{S}^{\prime}} \frac{d t}{a(t)}+\int_{t_{S c h u i S}^{\prime}}^{t_{S c h u ̈ S}} \frac{d t}{a(t)} .
\end{aligned}
$$

But since $a(t)$ varies significantly only on time intervals of cosmological nature

$$
\int_{t_{S}}^{t_{S}^{\prime}} \frac{d t}{a(t)} \simeq \frac{t_{S}^{\prime}-t_{S}}{a\left(t_{S}^{\prime}\right)} \int_{t_{S c h u ̈ S}}^{t_{S c h u}} \frac{d t}{a(t)} \simeq \frac{t_{S c h \ddot{u} S}-t_{S \operatorname{shüS} S}^{\prime}}{a\left(t_{S c h u ̈ S}^{\prime}\right)} .
$$

Then

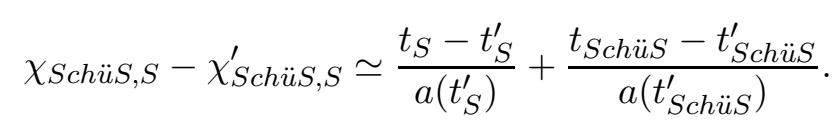

Equating the right hand sides of (118) and (123), one arrives at

$$
\frac{t_{S}-t_{S}^{\prime}}{a\left(t_{S}^{\prime}\right)}+\frac{t_{S c h \ddot{u} S}-t_{S c h u ̈ S}^{\prime}}{a\left(t_{S c h \ddot{u} S}^{\prime}\right)} \simeq \frac{\left(\varphi_{S c h u ̈ S}-\varphi_{S}\right)^{2}-\left(\varphi_{S c h u ̈ S}^{\prime}-\varphi_{S}\right)^{2}}{2\left(\chi_{L, S}^{-1}-\chi_{S c h \ddot{u}}^{-1}\right)} .
$$

Then one deduces an expression for $t_{S}-t_{S}^{\prime}$

$$
t_{S}-t_{S}^{\prime} \simeq a\left(t_{S}^{\prime}\right)\left[\frac{t_{S c h \ddot{u} S}^{\prime}-t_{S c h \ddot{u} S}}{a\left(t_{S c h \ddot{u} S}^{\prime}\right)}+\frac{\left(\varphi_{S c h \ddot{u} S}-\varphi_{S}\right)^{2}-\left(\varphi_{S c h \ddot{u} S}^{\prime}-\varphi_{S}\right)^{2}}{2\left(\chi_{L, S}^{-1}-\chi_{S c h \ddot{u}}^{-1}\right)}\right] .
$$

Hereafter are displayed the results for $\varphi_{S}\left(=\varphi_{S}^{\prime}\right)$ the deflexion angle, $M$ the mass of the cluster of galaxies (the lens) and $\Delta t=t_{S}-t_{S}^{\prime}$, the time delay, in the case of the lensed quasar SDSS J1004+4112 where [18, 19, 20]. ' \pm 0 ' stands for the central value, '+' and '-' stand respectively for the upper and the lower experimental limits.

$$
\begin{aligned}
\alpha^{\prime} & =5^{\prime \prime} \pm 10 \%, & \alpha & =10^{\prime \prime} \pm 10 \% \\
z_{L} & =0.68, & z_{S} & =1.734 .
\end{aligned}
$$

The cluster mass $M$ comes from a fitting: the angles $\varphi_{S}$ and $\varphi_{S}^{\prime}$ are calculated as a function of $M$ which is varied until the equality $\varphi_{S}^{\prime}=\varphi_{S}$ is satisfied. 
For the cosmological $\Lambda$, we take the experimentally favored $\Lambda=0.77 \cdot 3 \mathrm{am}^{-2} \pm 20 \%$. We have also considered the case without cosmological constant $\Lambda=0$ in table 4 ,

Table 1: Upper limit value of $\Lambda: \quad \Lambda=0.77 \cdot 3 \mathrm{am}^{-2}+20 \%$

\begin{tabular}{cccccccccc}
\hline$\alpha_{E} \pm 10 \%$ & \pm 0 & \pm 0 & \pm 0 & + & + & + & - & - & - \\
$\alpha_{E}^{\prime} \pm 10 \%$ & \pm 0 & + & - & \pm 0 & + & - & \pm 0 & + & - \\
$-\varphi_{S}\left[^{\prime \prime}\right]$ & 9.03 & 8.13 & 9.94 & 10.84 & 9.94 & $\mathbf{1 1 . 7 4}$ & 7.23 & $\mathbf{6 . 3 2}$ & 8.13 \\
$M\left[10^{13} M_{\odot}\right]$ & 1.80 & 1.98 & 1.62 & 1.98 & $\mathbf{2 . 1 8}$ & 1.78 & 1.62 & 1.78 & $\mathbf{1 . 4 6}$ \\
$\Delta t[$ years $]$ & 9.76 & 9.18 & 10.25 & 12.35 & 11.81 & $\mathbf{1 2 . 7 7}$ & 7.38 & $\mathbf{6 . 7 4}$ & 7.91 \\
\hline
\end{tabular}

Table 2: Central value of $\Lambda: \quad \Lambda=0.77 \cdot 3 \mathrm{am}^{-2}$

\begin{tabular}{ccccccccccc}
\hline$\alpha_{E} \pm 10 \%$ & \pm 0 & \pm 0 & \pm 0 & + & + & + & - & - & - \\
$\alpha_{E}^{\prime} \pm 10 \%$ & \pm 0 & + & - & \pm 0 & + & - & \pm 0 & + & - \\
$\left.-\varphi_{S}{ }^{\prime \prime}{ }^{\prime}\right]$ & 9.97 & 8.98 & 10.97 & 11.97 & 10.97 & $\mathbf{1 2 . 9 7}$ & 7.98 & $\mathbf{6 . 9 8}$ & 8.98 \\
$M\left[10^{13} M_{\odot}\right]$ & 1.82 & 2.00 & 1.64 & 2.00 & $\mathbf{2 . 2 1}$ & 1.80 & 1.64 & 1.80 & $\mathbf{1 . 4 8}$ \\
$\Delta t[$ years $]$ & 9.72 & 9.14 & 10.19 & 12.28 & 11.76 & $\mathbf{1 2 . 6 8}$ & 7.35 & $\mathbf{6 . 7 3}$ & 7.87 \\
\hline
\end{tabular}

Table 3: Lower limit value of $\Lambda: \quad \Lambda=0.77 \cdot 3 \mathrm{am}^{-2}-20 \%$

\begin{tabular}{|c|c|c|c|c|c|c|c|c|c|}
\hline$\alpha_{E} \pm 10 \%$ & \pm 0 & \pm 0 & \pm 0 & + & + & + & - & - & - \\
\hline$\alpha_{E}^{\prime} \pm 10 \%$ & \pm 0 & + & - & \pm 0 & + & - & \pm 0 & + & - \\
\hline$-\varphi_{S}\left[{ }^{\prime \prime}\right]$ & 10.5 & 9.51 & 11.63 & 12.68 & 11.63 & 13.74 & 8.46 & 7.40 & 9.51 \\
\hline$M\left[10^{13} M_{\odot}\right]$ & 1. & 1.98 & 1 & 1 & 2 . & 1. & 1.62 & 1.79 & 1.46 \\
\hline$\Delta t[$ years $]$ & 9.53 & 8.97 & 9.98 & 12.03 & 11.53 & 12.41 & 7.21 & 6.60 & 7.72 \\
\hline
\end{tabular}

\begin{tabular}{cccccccccc}
\multicolumn{10}{c}{ Table 4: $\Lambda=0$} \\
\hline$\alpha_{E} \pm 10 \%$ & \pm 0 & \pm 0 & \pm 0 & + & + & + & - & - & - \\
$\alpha_{E}^{\prime} \pm 10 \%$ & \pm 0 & + & - & \pm 0 & + & - & \pm 0 & + & - \\
$-\varphi_{S}\left[^{\prime \prime}\right]$ & 11.86 & 10.67 & 13.05 & 14.23 & 13.05 & $\mathbf{1 5 . 4 2}$ & 9.49 & $\mathbf{8 . 3 0}$ & 10.67 \\
$M\left[10^{13} M_{\odot}\right]$ & 1.68 & 1.84 & 1.51 & 1.84 & $\mathbf{2 . 0 3}$ & 1.66 & 1.51 & 1.66 & $\mathbf{1 . 3 6}$ \\
$\Delta t[$ years $]$ & 8.70 & 8.20 & 9.10 & 10.97 & 10.53 & $\mathbf{1 1 . 3 0}$ & 6.59 & $\mathbf{6 . 0 4}$ & 7.05 \\
\hline
\end{tabular}




\section{Conclusion}

In this paper, we have computed the time delay caused by a spherical mass, a cluster of galaxies, in presence of a cosmological constant $\Lambda$ using the Einstein-Straus solution, which is the appropriate framework for taking into account the precession of the observer and the effect of the other masses of the universe in the form of a homogeneous isotropic dust, the observer being taken comoving with the dust. We have applied our results to the lensed quasar SDSS J1004+4112. We have computed the time delay between the images $\mathrm{C}$ and $\mathrm{D}$ of the quasar, which are the most aligned images with the lens, and obtained results compatible with the lower bound given by Fohlmeister [21. Our predictions of the time delay range from 6 to 13 years.

It is worthwhile to compare our results with those of previous computations performed by Schücker and Zaimen [17] and by Kawano and Oguri [22. Schücker and Zaimen computed the time delay in the framework of the Kottler solution and obtained predictions ranging from 13 to 28 years, using however a different mass of the lens. On the other hand, Kawano and Oguri obtained a time delay of 10 years.

In addition to the hypothesis of sphericity we have made in our calculations an additional assumption: we have supposed implicitly that the photons don't penetrate the mass distribution region, since we have used only the exterior Kottler solution inside the vacuole. It is then worthwhile to repeat the calculations taking into account that the photons can penetrate the interior of the mass distribution, where an interior Kottler solution must be used [23].

\section{Acknowledgment}

This work was supported by Le Ministère de l'Enseignement Supérieur et de la Recherche Scientifique of Algeria under grant D00920090096.

\section{References}

[1] W. Rindler and M. Ishak, The Contribution of the Cosmological Constant to the Relativistic Bending of Light Revisited, Phys. Rev. D76 043006 [arXiv:0709.2948 [astro-ph]] (2007).

[2] M. Sereno, On the influence of the cosmological constant on gravitational lensing in small systems., Phys. Rev. D77, 043004 arXiv:0711.1802 [astro-ph]] (2008)

[3] M. Sereno, The role of Lambda in the cosmological lens equation. arXiv:0807.5123 [astro$\mathrm{ph}]]$

[4] T. Schücker, Cosmological constant and lensing. [arXiv:0712.1559 [astro-ph]], Gen. Relativ. Gravit. DOI:10.1007/s10714-008-0652-2

[5] T. Schücker, Strong lensing with positive cosmological constant. arXiv:0805.1630 [astroph]].Moriond Proceedings Cosmology (2008)

[6] H. Miraghaei, M. Nouri-Zonoz, Classical tests of general relativity in the Newtonian limit of Schwarzschild-de-Sitter spacetime. [arXiv:0810.2006 [gr-qc]]

[7] R. Kantowski, B. Chen and X. Dai, Gravitational lensing corrections in flat $\Lambda C D M$ cosmology. [arXiv:0909.3308v1 [astro-ph.CO]] 
[8] I.B. Khriplovich, A.A. Pomeransky, Does cosmological term influence gravitational lensing? arXiv:0801.1764 [gr-qc]]

[9] M. Park, Rigorous approach to the gravitational lensing. [arXiv:0804.4331 [astro-ph]]

[10] G.W. Gibbons, C.M. Warnick, M.C. Werner, Light-bending in Schwarzschild-de-Sitter: projective geometry of the optical metric. [arXiv:0808.3074 [gr-qc]]

[11] F. Simpson, J.A. Peacock, A.F. Heavens, On lensing by a cosmological constant. arXiv:0809.1819 [astro-ph]]

[12] T. Schücker, Strong lensing in the Einstein-Straus solution. Gen Relativ Gravit (2009) 41:1595-1610 DOI:10.1007/s10714-008-0731-4

[13] A. Einstein, E.G. Straus, The influence of the expansion of space on the gravitation fields surrounding the individual star. Rev. Mod. Phys. 17, 120 (1945), 18, 148 (1946)

[14] E. Schücking, Das Schwarzschildsche Linienelement und die Expansion des Weltalls. Z. Phys. 137, 595 (1954)

[15] M. Ishak, W. Rindler, J. Dossett, More on Lensing by a Cosmological Constant. arXiv:0810.4956 [astro-ph]]

[16] R. Balbinot, R. Bergamini, A. Comastri, Solution of the Einstein-Straus problem with a Lambda term. Phys. Rev. D38, 2415 (1988)

[17] T. Schücker, N. Zaimen, Cosmological constant and time delay. A\&A 484, 103 arXiv:0801.3776 [astro-ph]] (2008)

[18] N. Inada et al., [SDSS Collaboration], A Gravitationally Lensed Quasar with Quadruple Images Separated by 14.62 Arcseconds, Nature 426, 810 arXiv:astro-ph/0312427] (2003)

[19] M. Oguri et al., [SDSS Collaboration], Observations and Theoretical Implications of the Large Separation Lensed Quasar SDSS J1004+4112. Astrophys. J. 605, 78 arXiv:astro-ph/0312429 (2004)

[20] N. Ota et al., Chandra Observations of SDSS J1004+4112: Constraints on the Lensing Cluster and Anomalous X-Ray Flux Ratios of the Quadruply Imaged Quasar. Astrophys. J. 647, 215 [arXiv:astroph/0601700] (2006)

[21] J. Fohlmeister et al., The Rewards of Patience: An 822 Day Time Delay in the Gravitational Lens SDSS J1004+4112. arXiv:0710.1634 [astro-ph]] (2007)

[22] Y. Kawano and M. Oguri, Time delays for the Giant Quadruple Lensed SDSS J1004+4112: Prospects for Determining the Densityn Profile of the Lensing Cluster [arXiv:0601149v1 [astro-ph]] (2006)

[23] T. Schücker, Lensing in an interior Kottler solution arXiv:0903.2940 [astro-ph]] (2009) 